\title{
Multiple trans QTL and one cis-regulatory deletion are associated with the differential expression of cone opsins in African cichlids
}

\author{
Sri Pratima Nandamuri®, Matthew A. Conte and Karen L. Carleton ${ }^{*}$
}

\begin{abstract}
Background: Dissecting the genetic basis of phenotypic diversity is one of the fundamental goals in evolutionary biology. Despite growing evidence for gene expression divergence being responsible for the evolution of complex traits, knowledge about the proximate genetic causes underlying these traits is still limited. African cichlids have diverse visual systems, with different species expressing different combinations of seven cone opsin genes. Using opsin expression variation in African cichlids as a model for gene expression evolution, this study aims to investigate the genetic architecture of opsin expression divergence in this group.

Results: Results from a genome-wide linkage mapping on the $F_{2}$ progeny of an intergeneric cross, between two species with differential opsin expression show that opsins in Lake Malawi cichlids are controlled by multiple quantitative trait loci (QTLs). Most of these QTLs are located in trans to the opsins except for one cis-QTL for SWS1 on LG17. A closer look at this major QTL revealed the presence of a 691 bp deletion in the promoter of the SWS1 opsin (located $751 \mathrm{bp}$ upstream of the start site) that is associated with a decrease in its expression. Phylogenetic footprinting indicates that the region spanning the deletion harbors a microRNA miR-729 and a conserved noncoding element (CNE) that also occurs in zebrafish and other teleosts. This suggests that the deletion might contain ancestrally preserved regulators that have been tuned for SWS1 gene expression in Lake Malawi. While this deletion is not common, it does occur in several other species within the lake.
\end{abstract}

Conclusions: Differential expression of cichlid opsins is associated with multiple overlapping QTL, with all but one in trans to the opsins they regulate. The one cis-acting factor is a deletion in the promoter of the SWS1 opsin, suggesting that ancestral polymorphic deletions may contribute to cichlid's visual diversity. In addition to expanding our understanding of the molecular landscape of opsin expression in African cichlids, this study sheds light on the molecular mechanisms underlying phenotypic variation in natural populations.

Keywords: QTL, Cichlid, Opsin, Cis-regulation, SWS1

\section{Background}

Deciphering the genetic mechanisms underlying phenotypic diversity has long been at the forefront of evolutionary biology research. Much of what we know about the molecular basis for phenotypic variation comes from studies in classic model systems such as yeast and Drosophila $[1-5]$. This work has, without any doubt, vastly advanced

\footnotetext{
* Correspondence: kcarleto@umd.edu

Department of Biology, University of Maryland, 1210 Biology / Psychology Bldg \#144, College Park, MD 20742, USA
}

our understanding of trait evolution. We now know that the emergence of novel phenotypes can result from both mutations in coding sequences that alter the structure or function of the proteins, or by mutations in the regulatory sequences that modify the spatial and temporal expression patterns of genes. There have been animated debates in the literature on the importance of coding sequence versus regulatory mutations and the relative contribution of cis versus trans regulatory mutations in bringing about phenotypic change [6-9]. Although theory predicts that

(c) The Author(s). 2018 Open Access This article is distributed under the terms of the Creative Commons Attribution 4.0 International License (http://creativecommons.org/licenses/by/4.0/), which permits unrestricted use, distribution, and 
cis-regulatory mutations must predominate owing to their highly specific effects and reduced pleiotropy, results from actual studies are often conflicting [10-12]. Adding to the confusion is the fact that some of these studies were performed using inbred wild type and mutant lab raised individuals. Consequently, knowledge about the genetic processes responsible for phenotypic divergence in natural populations has been severely limited. However, recent advances in cost effective sequencing methods allow us to unravel the mechanistic basis for natural adaptation by combining sequence information with traditional quantitative trait loci mapping and candidate gene approaches. Such methods have recently been employed to uncover skeletal appendage patterning in sticklebacks, eye loss in Astyanax, feathered feet in pigeons and coat coloration in beach mice, among others [13-17]. Yet a coherent understanding of the relative roles of cis- versus trans- regulatory changes requires more studies representing a broader set of traits in naturally-occurring populations.

Vertebrate opsins include four main cone opsin families - SWS1, SWS2, RH2 and LWS as well as the RH1 family found in rods [18-21]. Experimental work in a variety of model systems has identified key factors governing photoreceptor differentiation and opsin expression [22, 23]. These studies have identified that opsins are controlled both by cis-factors and trans-acting elements. For example, studies in humans have shown that opsin genes are controlled by cis-enhancers located upstream of the genes [24]. Similarly, work in mice has shown that Thyroid Hormone Receptor Beta (THR $\beta$ ) transcription factor specifies photoreceptors to a M-cone (LWS family) fate [25]. Nonetheless, these studies fail to present a complete understanding of the molecular mechanisms underlying differential opsin expression, simply because model organisms such as mice and humans do not express representatives from all cone families. Owing to gene duplication events, fish typically have a more complex set of cones that are members of the four prototypical vertebrate cone classes [26]. This provides ample opportunity to study genetic factors underlying the specification of opsins that are usually absent in many other vertebrates, such as SWS1. In fact, separate studies in zebrafish and trout have identified that SWS1 opsin is downregulated by THR $\beta$ and upregulated by $T B X 2 B$ respectively $[27,28]$. However, no studies have yet been done to examine variable SWS1 opsin expression between closely related species in either trout or zebrafish and mechanisms driving the differential expression of SWS1 opsin largely remain unknown.

African cichlids are a particularly promising system to study the genetic basis of phenotypic diversity [29-31]. This highly speciose group of freshwater fish inhabit rift lakes throughout Africa, most notably in the three great lakes- Malawi, Victoria and Tanganyika [32]. Incredible bursts of parallel adaptive radiations have occurred in each of these lakes, making cichlids a classical example of explosive speciation. [33-35]. Just in Lake Malawi, more than 500 species have arisen in the last few million years following multiple repeated colonization and hybridization events [36, 37]. Cichlids are amenable for studying the genetics of adaptive traits for three main reasons. First, different species and even genera of cichlids can interbreed to produce fertile hybrids [38-40]. This makes mapping of traits for intraspecific and even interspecific comparisons possible. Second, owing to their recent divergence, key polymorphisms are likely to be shared between species [41]. Besides enabling us to find causative mutations, inferences about the evolutionary history of the trait can be easily drawn. Third, well-developed genomic resources are available for cichlids, with complete genomes for the riverine ancestor Tilapia and representative species from each of the three great lakes [42].

Within Lake Malawi, cichlids exhibit incredible diversity for a variety of anatomical, physiological and behavioral traits [43-46]. Closely related species often occupy different habitats, have varied coloration patterns, display numerous feeding strategies and breeding behaviors. For this study, we focus on the diversity of their visual systems, particularly in terms of the genes responsible for color discrimination, the cone opsins. All African cichlid species have seven cone opsin genes in their genomes, each maximally sensitive to a different part of the light spectrum [47]. These include three single cone opsins- SWS1(ultraviolet-sensitive), $S W S 2 B$ (violet-sensitive), $S W S 2 A$ (blue-sensitive), and four double cone opsins- $R H 2 B$ (blue-green-sensitive), RH2A-alpha and RH2A-beta (green-sensitive), and LWS (red-sensitive). While the SWS1 opsin in cichlids is located on LG17 (linkage group 17), the remaining opsins form two tandem arrays (SWS2A-SWS2B-LWS and RH2B-RH2A $\alpha-R$ $H 2 A \beta)$ separated by $30 \mathrm{Mb}$ on LG5 [48].

In Lake Malawi cichlids, large variation in visual sensitivities both within and between species has been shown to be mediated not by coding sequence changes but by alterations in the expression of the cone opsin genes [49-51]. As adults, species from Lake Malawi express different subsets of the seven genes, leading to dramatic differences in their visual sensitivities $[39,52]$. Besides being important for foraging, predator avoidance and habitat- preference, vision is key for cichlid fitness playing a major role in mate choice and courtship [53-57]. In most species, the males are colorful while females are drab and brown. During breeding season, colorful dominant males display aggressive mating behaviors and get visually selected by choosy females to spawn the next generation $[58,59]$. Sister taxa in cichlids often differ in 
visual sensitivities but not in morphology or behavior. Therefore, it has been hypothesized that the differences in visual capabilities due to variation in opsin gene expression may contribute significantly to the rapid speciation within this group [60, 61].

A genome-wide mapping study using hybrids from an intergeneric cross between Tramitochromis intermedius and Aulonocara baenschi (TA cross) was previously carried out in our lab to identify genomic regions associated with the differential expression of opsins [62]. Results from that study identified 11 QTL for 5 cone opsins segregating in the cross- 2 loci each for single cone opsins (SWS2A and SWS2B- LG5, LG23) and 7 loci for double cone opsins (RH2B, RH2A, LWS- LG1, LG5, LG10, LG15 and LG23) Here, $R H 2 A \alpha$ and $R H 2 A \beta$ are more than $99 \%$ identical and thus have been merged together as $R H 2 A$. Most of these QTLs are positioned on chromosomes that do not contain the opsin genes they are linked to, indicating that the cone opsins on LG5 are not coordinated by cis-regulatory regions adjacent to the opsin, but are regulated by trans-loci located elsewhere in the genome. Even the QTLs on LG5 are located distantly from the opsin genes, separated by $\sim 720 \mathrm{~kb}$, and conservatively classified to be functioning in trans. More importantly, since neither of the parental species from the cross had SWS1 expression, the basis of SWS1 differential expression could not be determined.

To find genetic loci controlling the differential expression of SWS1 opsin, the current study relies on a new hybrid cross between two Lake Malawi species- Aulonocara baenschi (negligible SWS1 expression) and Metriaclima mbenji (high SWS1 expression, MA cross) [63]. In cichlids, the SWS1 gene is a single copy opsin on LG17 separated from the rest of the 6-opsin cluster [48]. Previously, we found an association between SWS1 expression and a micro-satellite marker located in the third intron of the opsin gene, suggesting that differential expression of SWS1 opsin could be governed by cis-regulation [63]. However, this association was tested with just one marker and only in a subset of the $\mathrm{F}_{2}$.

Here, we use restriction-site associated DNA sequencing (RAD-Seq) and QTL mapping with markers across the whole genome, using $F_{2}$ progeny from the same cross to get a more complete picture of the genetic mechanisms governing SWS1 expression. We find three QTLs associated with the differential expression of SWS1- two in trans on LG14 and LG20, and one located in cis to the SWS1 opsin gene on LG17, indicating that the modulation of SWS1 opsin expression evolved through changes in both cis-regulatory sequences as well as trans-acting factors. Also, we identify additional loci whose segregation is associated with the differential expression of the other opsins (SWS2B, RH2B, RH2A and $L W S$ ). In addition to presenting a more detailed picture of the genetic architecture of opsin expression in cichlids, this study will lead to a more coherent understanding of the molecular mechanisms contributing to adaptive phenotypic diversity.

\section{Results \\ Opsin expression variation in the F2 hybrids}

As described in Nandamuri et al. [63], opsin expression measured by quantitative PCR varied considerably among the $157 \mathrm{~F}_{2}$ hybrids in the MA cross. Most opsins showed a dominant pattern of inheritance in the $F_{2}$ hybrids, with expression of $S W S 1, S W S 2 B$ and $R H 2 B$ leaning towards levels observed in $A$. baenschii $\mathrm{F}_{0}$, while that of $R H 2 A$ was closer to levels in $M$. mbenji $\mathrm{F}_{0}$. Consistent with expression in both the $\mathrm{F}_{0}$ parents, the $\mathrm{F}_{2}$ hybrids showed negligible levels of SWS2A expression. Expression of $L W S$ opsin in the double cones was transgressive with $\mathrm{F}_{2}$ hybrids showing higher expression than either of the parents of the cross. Since expression of each opsin in the cross is characterized as a fraction of total expression in single cones (SWS1, $S W S 2 B$, $S W S 2 A)$ and double cones $(R H 2 B, R H 2 A, L W S)$ respectively, and since most $\mathrm{F}_{2}$ hybrids show negligible SWS2A expression, SWS1 and SWS2B opsins become inversely correlated.

\section{RAD-Seq genotyping}

After removal of reads with ambiguous barcodes, Illumina sequencing of the two RAD-Seq libraries yielded a total of $\sim 185$ million reads for the $F_{2}$ hybrids. Around $97 \%$ of these reads ( 178 million) passed the quality filters and were retained for subsequent processing. This represented a total of $\sim 66,500$ SbfI restriction sites in the cichlid genome, corresponding to an average read depth of $17 x$ for each $F_{2}$ individual. Similarly, after filtering and barcode processing, the average read coverage in the M. mbenji $\mathrm{F}_{0}$ and A. baenschii $\mathrm{F}_{0}$ was $16 \mathrm{x}(\sim 1$ million reads) and 70x ( $\sim 4.6$ million reads) respectively. The low coverage in the $M$. mbenji $\mathrm{F}_{0}$ was due to the low-quality DNA. The RAD-Seq processing statistics for the $F_{2}$ and the $F_{0}$ individuals are provided in Additional file 1. In total, Stacks software identified 2370 RAD-Seq SNPs (single-nucleotide polymorphisms) following stringent cutoffs. After filtering to identify differentially fixed SNPs in the $\mathrm{F}_{0}$, anchoring to a unique location in the M. zebra genome and adherence to Hardy-Weinberg equilibrium, we retained a conservative set of 1217 SNPs for linkage map and QTL analysis. The FASTA sequences of the 1217 RAD markers are provided in Additional file 2. The total fraction of missing genotypes across all 1217 SNP markers and $157 \mathrm{~F}_{2}$ individuals was $4.5 \%$ while the frequency of each genotypic class was $26.1 \%$ homozygous for $M$. mbenji alleles 
(MM), 25.4\% homozygous for A. baenschi alleles (AA) and $48.5 \%$ heterozygous (MA).

\section{Linkage mapping and anchoring to M. zebra genome} A high-density linkage map comprising 22 linkage groups spanning $1558.1 \mathrm{cM}$ was assembled (Additional file 3: Figure S1). The average intermarker distance was $1.3 \mathrm{cM}$ and the maximum distance between consecutive markers was 20.9 cM. Previous karyotyping work has shown that a haploid cichlid genome is comprised of 22 chromosomes indicating that the linkage map obtained in this study is complete, with one linkage group per chromosome [48]. Also, the length of the map is comparable to previously published linkage maps constructed from other cichlid crosses with closely related species $[45,62,64]$. The linkage map anchored $662,849,923 \mathrm{bp}$, representing $69.3 \%$ of the $M$. zebra genome assembly. The linkage map as well as the phenotype and genotype information used for QTL analysis is provided in Additional file 4.

\section{QTL for single cone opsins}

Using quantitative trait locus mapping, we detected a total of nine significant QTL for single cone opsin expression- three for SWS1 (LG14, LG20 and LG17), three for $S W S 2 B$ (LG14, LG20 and LG17) and three for $S W S 2 A$ (all on LG23) (Fig. 1). However, the three $S W S 2 A$ QTL were likely an artifact of the multiple scan for two reasons: 1) the three loci on LG23 are in close proximity to each other and not separated by at least one genotyped marker. 2) the significant signal is driven by just 10 (of the $157 \mathrm{~F}_{2}$ ) individuals showing less than $2 \% S W S 2 A$ expression. Therefore, the SWS2A QTL were removed from subsequent analysis. Due to the nature of normalization in the single cones and the absence of SWS2A expression in most $\mathrm{F}_{2}$ hybrids, overlapping QTLs were obtained for both the SWS1 and $S W S 2 B$ opsins (Fig. 1). The LOD (logarithm of odds) scores, QTL intervals and percent variance in opsin expression (PVE) explained by each of the QTLs are provided in Table 1.

Genome-wide LOD threshold calculated at a significance of $P=0.05$ is 3.9 for both the SWS1 and $S W S 2 B$ opsins. For the SWS1 opsin, the QTL on LG17, spanning a region of $6.2 \mathrm{cM}$, is the most significant of the three QTL $(\mathrm{LOD}=17.97)$ (Fig. 2a). Coincidentally, the SWS1 opsin gene is located within this QTL interval, suggesting that the SWS1 expression is potentially cis-regulated. Additionally, this QTL has the largest effect on SWS1 expression, accounting for $31.65 \%$ of the variance in $\mathrm{F}_{2}$ expression. The two QTLs on LG14 $(\mathrm{LOD}=6.62)$ and LG20 (LOD $=8.58)$ are trans QTL explaining 9.8 and $13 \%$ variation in SWS1 expression respectively (Fig. 2a). The $S W S 2 B$ opsin is located on LG5. Therefore, all three QTL found in this study are located in trans. Similar to SWS1, LG17 (LOD = 17.1) is the largest contributor to $S W S 2 B$ expression, explaining $31.3 \%$ of the variance (Fig. 2b). Around 8.7 and $14.6 \%$ $\mathrm{F}_{2}$ expression variance is explained by the remaining QTL on LG14 $(\mathrm{LOD}=5.68)$ and LG20 (LOD = 9.04) respectively (Fig. $2 \mathrm{~b}$ ).

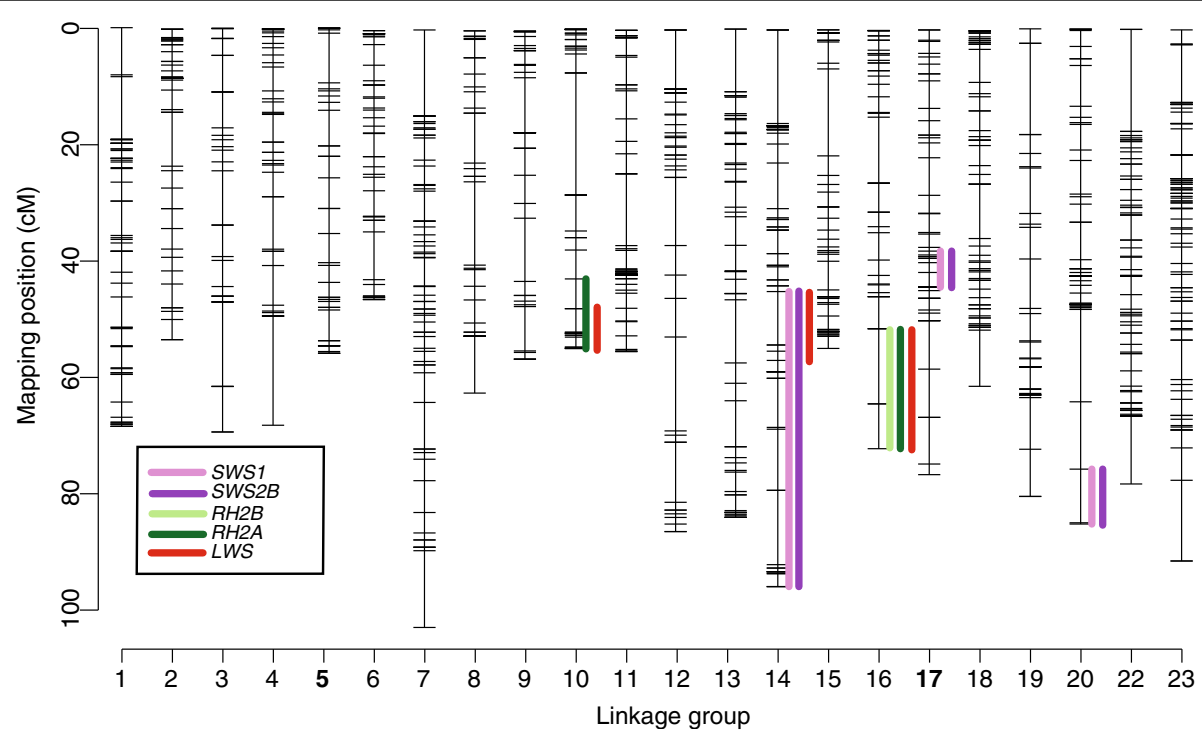

Fig. 1 Genome wide distribution of cone opsin QTL. Quantitative trait loci (QTL) mapping detected 12 intervals on 5 LGs that segregated for the differential expression of cone opsins between the two $F_{0}$ species. In the single cones, SWS1 and SWS2B have 3 co-localizing QTLs on LG14, LG17 and LG20. While RH2B in the double cones has only 1 QTL on LG16, RH2A and LWS share 2 QTL on LG10 and LG16. Single cones and double cones share 1 QTL on LG14. Each cone opsin QTL is indicated by a separate color depicted in the color legend. The cone opsins in cichlids are present on two chromosomes- LG5 (SWS2B, SWS2A, LWS, RH2B, RH2Aa, RH2AB) and LG17 (SWS1). The black lines represent RAD loci 
Table 1 Summary of QTL for single cone opsin expression in the MA cross

\begin{tabular}{lllllll}
\hline Opsin & LG & Location $(\mathrm{CM})$ & LOD & PVE & 95\% BI (cM) & 95\% BI (markers) \\
\hline SWS1 & 17 & 39.28 & 17.97 & 31.65 & $38.02-44.21$ & RAD_33279 to RAD_39835 \\
SWS1 & 20 & 81 & 8.58 & 13.04 & $75.64-85.07$ & RAD_22102 to RAD_52696 \\
SWS1 & 14 & 56.81 & 6.62 & 9.77 & $44.96-95.69$ & RAD_41335 to RAD_2689 \\
SWS2B & 17 & 39.28 & 17.09 & 31.3 & $38.02-44.21$ & RAD_33279 to RAD_39835 \\
SWS2B & 20 & 81 & 9.04 & 14.6 & $75.64-84.86$ & RAD_22102 to RAD_8560 \\
SWS2B & 14 & 92.5 & 5.68 & 8.72 & $44.97-95.69$ & RAD_41335 to RAD_2689 \\
\hline
\end{tabular}

LOD Logarithm of Odds, PVE Percentage of variance explained in the $\mathrm{F}_{2}, \mathrm{BI} 95 \%$ Bayes interval
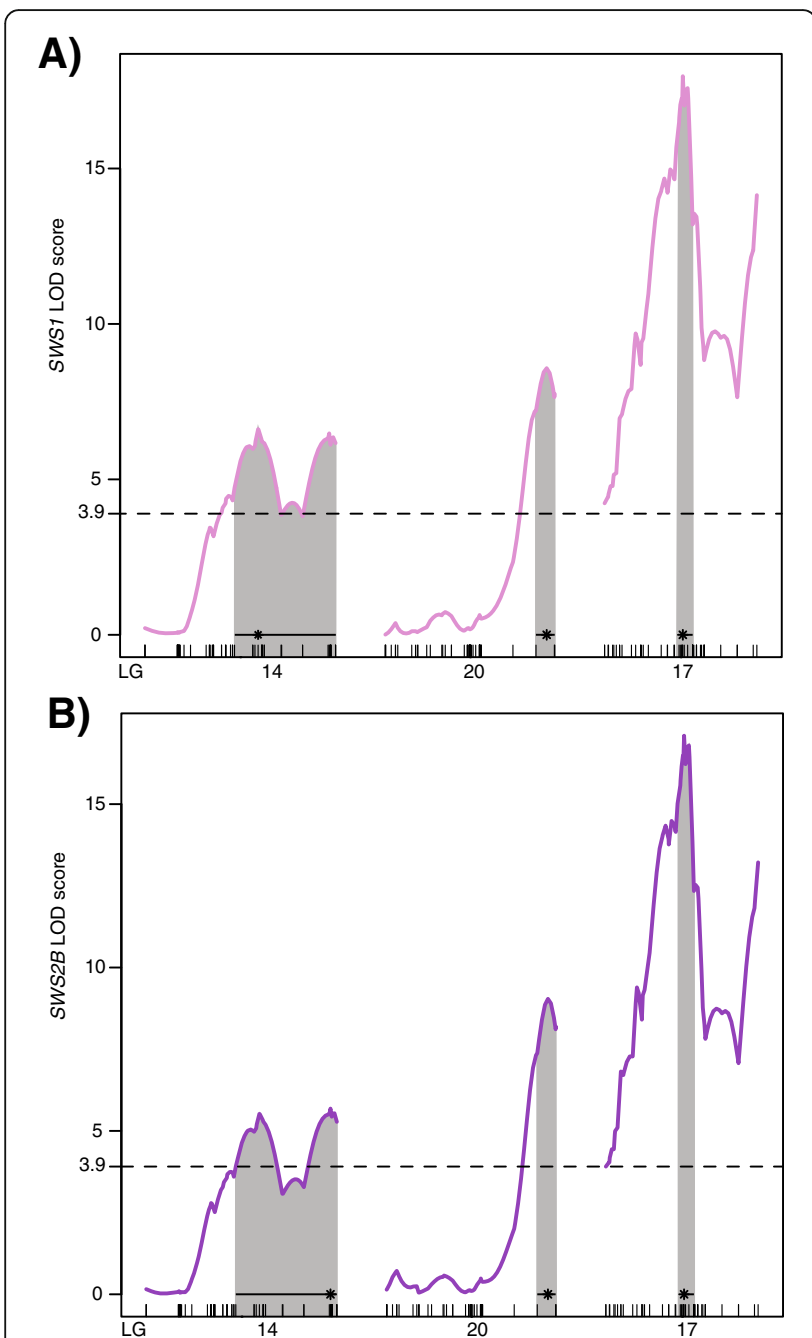

Fig. 2 QTL mapping for SWS1 and SWS2B opsins. LOD scores of significant QTL for single cone opsins. Horizontal dotted line indicated genome-wide significance threshold for both opsins at a significance $P=0.05$. The black line at the bottom of each QTL indicates the $95 \%$ Bayes credible interval. The asterisk denotes the location of the most significant marker (highest LOD score) in each QTL. a QTL for SWS1 expression on LG14, LG20 and LG17. The SWS1 opsin is also located in the Bayes interval of the QTL on LG17, 20 bp from the most significant marker. $\mathbf{b}$ QTL for SWS2B expression on LG14, LG20 and LG17
Effect plots for SWS1 show that the two QTLs on LG14 and LG17 are additive with higher SWS1 expression observed in the $F_{2}$ hybrids that are homozygous for the $M$. mbenji alleles $(\mathrm{MM}>\mathrm{MA}>\mathrm{AA})$ (Additional file 3: Figure S2A). However, the QTL on LG20 shows a dominant pattern of inheritance with both heterozygotes and $A$. baenschi homozygotes showing equal levels of low SWS1 expression $(\mathrm{MM}>\mathrm{MA}=\mathrm{AA})$ suggesting that the Aulonocara allele is a suppressor of $S W S 1$ expression (Additional file 3: Figure S2A). Effect plots for $S W S 2 B$ are identical except in the opposite direction (LG14 and LG17: $\mathrm{MM}<\mathrm{MA}<\mathrm{AA}$; $\mathrm{LG} 20: \mathrm{MM}<\mathrm{MA}=\mathrm{AA}$ (Additional file 3: Figure $\mathrm{S} 2 \mathrm{~B})$ ).

\section{QTL for double cone opsins}

The genome-wide LOD thresholds for $R H 2 B, R H 2 A$ and LWS opsins are 3.81, 3.91 and 3.78 respectively. For the double cone opsins, we identified a total of six significant QTL: one for $R H 2 B$ on LG16 (LOD = 4.97); two for $R H 2 A$ on LG10 (LOD = 6.37) and LG16 (LOD = 8.27); and three for LWS on LG14 (LOD =6.46), LG10 (LOD $=15.44)$ and LG16 (LOD = 4.18) (Figs. 1 and 3). All of these QTL are in trans to the double cone opsin genes that are located in two separate tandem arrays on LG5. Similar to the single cones, the normalization procedure leads to some degree of autocorrelations between the expression of the double cone opsins. As a result, we obtain overlapping QTLs for all the double cone opsins on LG16, and for RH2A and LWS on LG10 (Figs. 1 and 3). It is important to note that $S W S 1, S W S 2 B$ and $L W S$ opsins have one overlapping QTL on LG14 that is independent of the normalization, suggesting that genetic cross-talk exists between the single and double cones (Fig. 1).

The LOD scores, QTL intervals and percent variance in opsin expression (PVE) explained by each of the QTLs are provided in Table 2. The double cone opsin QTL on LG16 explain 13.56, 18.2 and $6.67 \%$ of the $F_{2}$ variance in $R H 2 B, R H 2 A$ and $L W S$ expression respectively. While the LWS QTL on LG10 is a major contributor accounting for $29.25 \%$ of the variance in the $F_{2}$ 


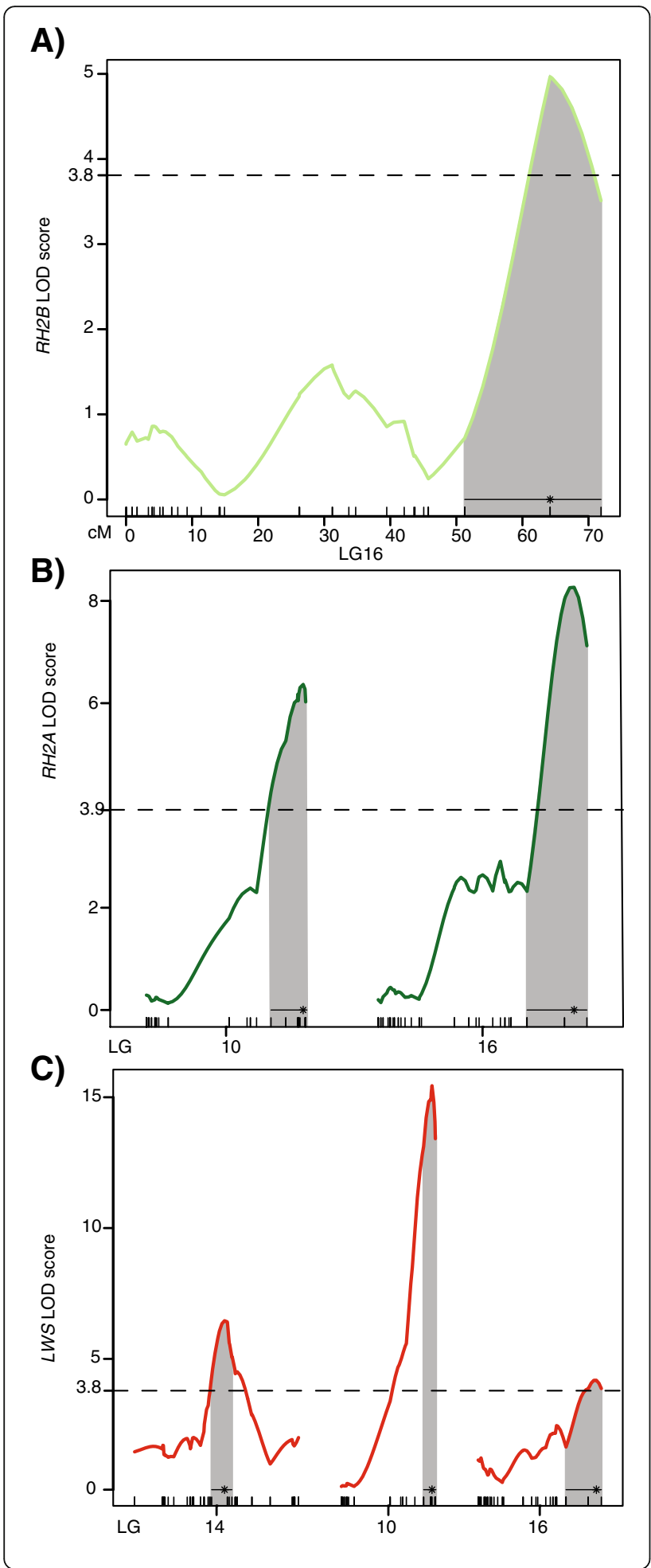

Fig. 3 QTL mapping for $R H 2 B, R H 2 A$ and LWS opsins. LOD scores of significant QTL for single cone opsins. Horizontal dotted line indicated genome-wide significance threshold for all opsins at a significance $P=0.05$. The black line at the bottom of each QTL indicates the 95\% Bayes credible interval. The asterisk denotes the location of the most significant marker (highest LOD score) in each QTL. a QTL for RH2B expression on LG16. b QTL for RH2A expression on LG10 and LG16. c QTL for LWS expression on LG14, LG10 and LG16

expression, the overlapping $R H 2 A$ QTL only explains $13.61 \%$ of its variation. Lastly, $10.65 \%$ variance in LWS expression is proportioned by the QTL on LG14.

Similar to the single cone opsin QTL on LG20, RH2B QTL on LG16 shows dominance, with equal levels of expression in $\mathrm{F}_{2}$ heterozygotes and $A$. baenschi homozygotes $(\mathrm{MM}<\mathrm{MA}=\mathrm{AA})$ (Additional file 3: Figure $\mathrm{S} 3 \mathrm{~A})$. While the expression of $R H 2 A$ opsin on LG10 is additive $(\mathrm{MM}<\mathrm{MA}<\mathrm{AA})$, heterozygous genotypes at LG16 have lower levels of $R H 2 A$ expression than either of the $M$. mbenji or A. baenschi homozygotes, indicating underdominance $(\mathrm{MM}>\mathrm{MA}<\mathrm{AA})$ (Additional file 3: Figure $\mathrm{S} 3 \mathrm{~B}$ ). Lastly, the three loci regulating LWS expression on LG14, LG10 and LG16 demonstrate underdominance $(\mathrm{MM}>\mathrm{MA}<\mathrm{AA})$, additivity $(\mathrm{MM}>\mathrm{MA}>\mathrm{AA})$ and overdominance $(\mathrm{MM}<\mathrm{MA}>\mathrm{AA})$ respectively (Additional file 3: Figure S3C).

\section{Candidate genes for opsin expression}

The QTL intervals on LGs 14, 20, 17, 16 and 10 each map to distinct regions of the $O$. niloticus genome, spanning on average $8 \mathrm{Mb}$ (The O. niloticus genome was used since the gene annotations were complete). Except for the cis-QTL on LG17, all the other trans-QTL contain numerous genes that potentially could contribute to the differential expression of the opsins. We have flagged certain genes as the most likely candidates based on their previous implication with opsin expression in the TA cross and/or association with photoreceptor function in other organisms (Table 3).

\section{SWS1 coding sequence comparison between $M$. mbenji} and $A$. baenschi

In both grandparents of the MA cross, $M$. mbenji and $A$. baenschi, the 5 exons of the SWS1 gene encode a 336-amino acid opsin protein that shows extensive sequence similarity to the SWS1 encoded by M. zebra (Fig. 4). However, the opsins differ from each other at 10 amino acid sites. We determined that 2 out of these 10 sites (corresponding to bovine rhodopsin sites 114 and 160 ) could potentially change the polarity of the amino acid and likely be spectrally tuning, thereby resulting in subtle distinction in the spectral sensitivity of the SWS1 opsin between the two species. 
Table 2 Summary of QTL for double cone opsin expression in the MA cross

\begin{tabular}{lllllll}
\hline Opsin & LG & Location (cM) & LOD & PVE & 95\% Bl (cM) & 95\% BI (markers) \\
\hline RH2B & 16 & 64.19 & 4.97 & 13.56 & $51.27-71.89$ & RAD_10047 to RAD_19271 \\
RH2A & 16 & 67.5 & 8.27 & 18.2 & $51.27-71.89$ & RAD_10047 to RAD_19271 \\
RH2A & 10 & 54 & 6.37 & 13.61 & $42.9-54.86$ & RAD_14019 to RAD_39775 \\
LWS & 10 & 52.91 & 15.44 & 29.25 & $48.02-54.63$ & RAD_45540 to RAD_1385 \\
LWS & 14 & 52.5 & 6.46 & 10.65 & $44.97-56.81$ & RAD_41335 to RAD_49138 \\
LWS & 16 & 69 & 4.18 & 6.66 & $51.27-71.89$ & RAD_10047 to RAD_19271 \\
\hline
\end{tabular}

LOD Logarithm of Odds, PVE Percentage of variance explained in the $F_{2}, B 195 \%$ Bayes interval

\section{Cis-regulatory elements important for SWS1 expression}

To test for causative regulatory mutations for the differential expression of SWS1 opsin, we sequenced approximately $2 \mathrm{~kb}$ of the promoter of the gene in both $M$. mbenji and $A$. baenschi $\mathrm{F}_{0}$ using overlapping primer sets. We found a $691 \mathrm{bp}$ deletion located $751 \mathrm{bp}$ upstream of the start codon of the SWS1 gene in A. baenschi (low SWS1 expression) that was absent in the promoter of $M$. mbenji (high SWS1 expression) (Fig. 5). To test if this region was variable in other cichlid species, we sequenced 17 species from Lake Malawi with previously characterized opsin profiles. Although nine of the 17-species panel had low/negligible levels of SWS1 expression, none of the species in the panel had the deletion in the SWS1 promoter. We further analyzed this region for the presence/absence of the deletion in 29 additional species from Lake Malawi by size using gel-electrophoresis. Only one of the 29 species tested, Placidochromis milomi, shared the promoter deletion. Additionally, examination of cichlid genomes sequenced by Malinsky et al. [65] revealed that the same deletion was present in three species- Aulonocara stuartgranti, Placidochromis milomi and Trematocranus placodon. All three of these species have no/low SWS1 expression. While the Trematocranus individual from our lab did not have the deletion, the sample from Malinsky et al. [65] did, suggesting within-species variation. This kind of individual variation is not unexpected and has been previously documented in Malawi cichlids [65]. Finally, neither of the representative cichlid species from Lake Tanganyika and Lake Victoria nor the two riverine ancestors had the deletion. All sampled species along with their opsin expression profiles are provided in Additional file 5.

We used mVISTA to perform phylogenetic footprinting of the SWS1 promoter of M. zebra (lacking the deletion) with orthologous regions from several other teleosts (tilapia, stickleback, medaka), including zebrafish. Our results show that the promoter of the SWS1 gene bears a high degree of conservation for two well described elements- a miRNA (encoded on the strand opposite to that of the SWS1 opsin) and a conserved non-coding element (CNE) (Fig. 6), both of which are located within the region spanning the deletion [66, 67].

Using mirBase, we found that the miRNA bears a high level of conservation with annotated zebrafish miRNA dre-miR-729 (See Additional file 6). It is conserved across teleosts and work in medaka has shown that it is coexpressed with SWS1 opsin in the same photoreceptors and that both the miRNA and SWS1 opsin are bidirectionally transcribed using the CNE element [67].

Table 3 Putative candidate genes for the differential expression of opsins in all QTLs except LG17

\begin{tabular}{|c|c|c|c|c|}
\hline Gene & Location & Accession number & Function & $\begin{array}{l}\text { Reference } \\
\text { (if available) }\end{array}$ \\
\hline Paired box protein Pax3B & LG14:3942202 & XM_005461712.1 & Involved in neural development. & {$[102]$} \\
\hline Zinc finger protein neurod4 & LG14:4686207 & XM_005454306.1 & $\begin{array}{l}\text { Regulates the proliferation of photoreceptor } \\
\text { progenitors }\end{array}$ & [81] \\
\hline Max-binding protein MNT & LG14: 14694887 & XM_005463727.1 & $\begin{array}{l}\text { Transcriptional repressor associated with retinitis } \\
\text { pigmentosa. }\end{array}$ & \\
\hline T-box transcription factor TBX2b & LG10:5759829 & XM_005471922.1 & UV photoreceptor specification in zebrafish & [27] \\
\hline Retinal guanylyl cyclase 2 & LG10:8655640 & XM_003456801.2 & $\begin{array}{l}\text { Expressed with the cone cells in the retinas of } \\
\text { zebrafish }\end{array}$ & [103] \\
\hline GPCR Kinase 1 & LG10:8655640 & XM_003456840.2 & $\begin{array}{l}\text { Phosphorylates rhodopsin and leads to its } \\
\text { deactivation }\end{array}$ & [82] \\
\hline Nuclear Receptor Subfamily 1D1 & LG20:25027615 & XM_005478044.1 & $\begin{array}{l}\text { Interacts with NR2E3 and plays critical role in } \\
\text { photoreceptor development }\end{array}$ & [83] \\
\hline
\end{tabular}




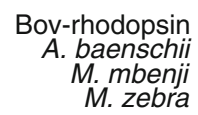

Bov-rhodopsin

A. baenschil M. mbenji M. zebra
VTVQHKKLRTPLNYILLNLAVADLFMVFGGFTTTLYTSLHGYFVFGPTGCNLEGFFATLG ATMKYKKLRVPLNFILVNISFSGFIFVTFSVSOVFLASMRGYFFLGHTLCALEGAVGSVA ATMKYKKLRVPLNFILVNISFSGFIFVTFSVSOVFLASMRGYYFLGHTLCALESAVGSVA ATMKYKKLRVPLNF ILVNISFSGF IFVTFSVSOVFLASMRGYYFLGHTLCALESAVGSVA

*

GEIALWSLVVLAIERYVVVCKPMSNFRFGENHAIMGVAFTWVMALACAAPPLVGWSRYIP GLVTAWSLAVLSFERYLVICKPFGAFKFGSNHALAAVAFAWFMGVGCACPPFFGWSRYIP GLVTAWSLAVISFERIVICKPFGAFKEGSNHALAAVAFTWFMGIGCACPPFFGWSRYI GLVTAWSLAVLSFERYLVICKPFGAFKFGSNHALAAVAFTWFMGIGCACPPFFGWSRYIP

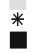

EGMQCSCGIDYYTPHEETNNESFVIYMFVVHFIIPLIVIFFCYGQLVFTVKEAAAQQQOES EGLGCSCGPDWYTHNEQYNTTSY IHFLMVTCF IMPLS I I IFCYSOLLGALRAVAAQQAES EGLGCSCGPDWYTHNEOYNTTSYTHFLMVTCFI I PLS I I IFCYSOLLGALRAVAAOOAES EGLGCSCGPDWYTHNEQYNTTSYTHFLMVTCFI IPLSI I IFCYSOLLGALRAVAAOOAES

ATTOKAEKEVTRMVI IMVIAFLICWLPYAGVAFY IFTHOGSDFGP IFMTIPAFFAKTSAV ASTOKAEKEVSRMIIVMVGSFVTCYGPYALAALYFAYSTDENKDYRLVTIPAFFSKSACV ASTOKAFKEVSRMI IVMVGSFVTCYGPYALAALYFAYSTDENKDYRLVTIPAFFSKSACV ASTQKAEKEVSRMIIVMVGSFVTCYGPYALAALYFAYSTDENKDYRLVTIPAFFSKSACV
60

120

180

240

300

YNPVIY IMMNKQFRNCMVTTLCCGKNPLGDDEASTTVSKTETSQVAPA YNPLIYVFMNKOFNGCIMEMVFGK-TMDESSEVS---TKTEVSTAS-YNPLIYVFMNKOVSTRIMEMVFGK-TMDESSEVS---TKTEVSTAS-YNPLIYVFMNKOFNGCIMEMVFGK-TMDESSEVS---TKTEVSTAS--

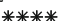

Fig. 4 Comparison of coding sequences between A. baenschi and M. mbenji. Alignment of coding sequence of SWS1 opsin from the two $F_{0}$ of the cross, $M$. zebra and Bovine rhodopsin. Gray bars indicate amino acid residues present in the retinal binding pocket that are predicted to be spectrally tuning. 10 amino acids (indicated by asterisks) are different between M. mbenji and A. baenschi. 2/10 sites (indicated by the black square) represent substitutions that cause a change in the physical properties of the site. These two sites could alter the sensitivity of the SWS1 opsin in A. baenschi relative to M. mbenji. Amino acids are numbered according to bovine rhodopsin

We used the 3' UTRs of all genes in the Tilapia genome (O_niloticus_UMD1, GCA_001858045.2), to find prospective targets for miR-729. Out of the $\sim 28,000$ genes predicted in the cichlid genome, we found that the miR-729 could potentially interact with 1024 genes.
Subsequent filtering of this list based on conserved roles in photoreceptor development and regulation enabled us to identify six top candidate genes that could play key roles in modulating opsin expression through their regulation by miR-729 (Table 4). Similarly, in silico analysis

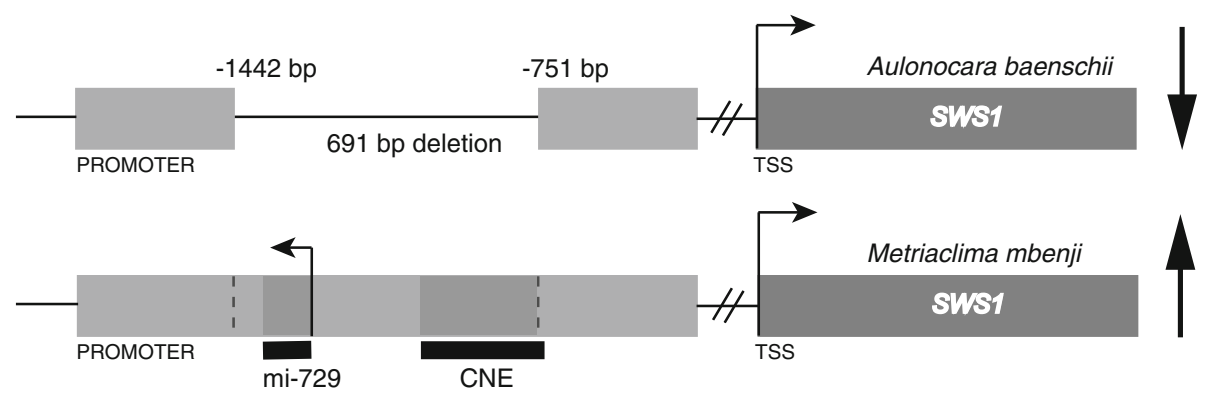

Fig. 5 Cis-regulatory mutation in the SWS1 opsin regulates its expression. Schematic representation of the promoter of the SWS1 gene in both the $F_{0}$ of the cross. Sequencing revealed a 691 bp deletion located 751 bp upstream of the translation start site (TSS) of the opsin in A. baenschi (low SWS1 expression) that is absent in the promoter of M. mbenji (high SWS1 expression). The region spanning the deletion comprises a miRNA miR-729 (transcribed on the strand opposite to that of the opsin) and a conserved non-coding element (CNE). Expression of the SWS1 opsin in both the species is denoted by the black arrow located to the right of the gene 


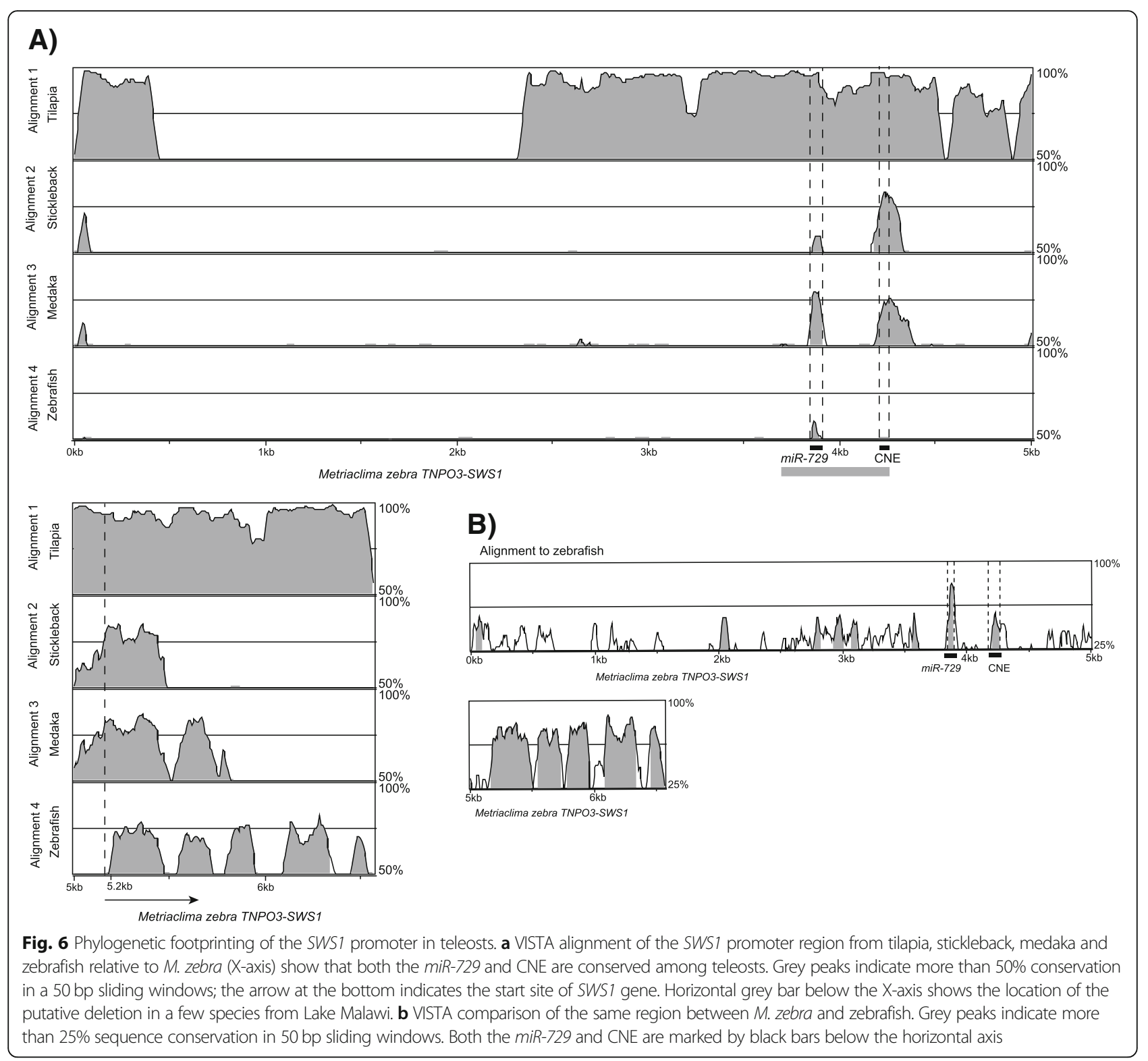

of the CNE predicted several putative binding sites for transcription factors connected with photoreceptor development in other systems (Table 4).

\section{Discussion}

In order to unravel the molecular mechanisms underlying the visual diversity in Lake Malawi cichlids, we utilized an intergeneric cross (M. mbenji X A. baenschi) and reduced representation sequencing of the $\mathrm{F}_{2}$ genome to find loci associated with cone opsin expression. We find a total of 12 QTL linked to the expression of six cone opsins between the two species, corresponding to five unique genomic loci. It is possible that the modest size of our $F_{2}$ population restricted our power to detect additional small QTLs with minor effects and also might have led to an overestimation of the variances explained by the individual QTL [68]. Although the absolute variances for each QTL might be inflated, we hypothesize that the relative magnitudes of the identified QTL for each opsin are most likely accurate. Future fine-mapping with additional $F_{2}$ individuals will help provide more exact estimates of the absolute variances explained by each QTL. Since our RAD-Seq approach for detecting QTLs relies on finding alternately fixed SNPs between the grandparents of the cross, it is also plausible that additional QTL might not have been identified.

\section{Regulation of SWS1 opsin in Lake Malawi cichlids}

QTL mapping revealed three QTLs associated with the differential expression of SWS1- two in trans on LG14 
Table 4 Potential targets of miR-729 and transcription factors (TFs) with putative binding sites in the CNE

\begin{tabular}{ll}
\hline Putative targets of miR-729 & Potential binding TFs in the CNE \\
\hline 1. Retinoic acid receptor alpha-A & 1. Cone-rod homeobox \\
2. Retinoic acid receptor RXR-gamma-B & 2. Neural Leucine Zipper \\
3. Cone-rod homeobox & 3. Orthodenticle Homeobox \\
4. Thyroid hormone receptor alpha & 4. Microphthalmia-Associated \\
5. Homeobox protein SIX2 & Transcription Factor \\
6. Thyroid hormone receptor- & 5. Retina and Anterior Neural \\
associated protein 3 & Fold Homeobox \\
& 6. Visual System Homeobox \\
& 7. Ventral Anterior Homeobox
\end{tabular}

and LG20, and one located in cis to the SWS1 opsin gene on LG17. The cis-QTL is the largest of the three QTL spanning a $7 \mathrm{Mb}$ region on LG17 with the SWS1 opsin gene located $20 \mathrm{bp}$ from the most significant marker. Finding a QTL located in cis to the SWS1 opsin is very surprising because results from this study as well as our previous TA cross suggest that all other opsins are regulated by multiple trans acting loci [62].

Comparison of the SWS1 protein sequences revealed two amino acid differences between the parental species of the MA cross that could potentially alter the maximal sensitivity of the opsin. Based on evidence from previous site-directed mutagenesis analysis and microspectrophotometry (MSP) studies, we theorize that the shift would be very small, altering the sensitivity of the opsin by just a few $(\sim 10)$ nanometers $[50,69]$. However, this inference is not without caveats and exact sensitivity of SWS1 opsin in both parental species can only be determined by experimental verification [70]. Regardless, it is safe to say that the sensitivity of the visual system would be affected negligibly by the coding sequence changes, especially when compared to the substantial shift due to the change in opsin expression from SWS1 to $S W S 2 B$ ( $65 \mathrm{~nm})$ [52]. While adult $A$. baenschi have no appreciable expression of SWS1 in their retinas, M. mbenji show high levels of expression [63]. We postulate that the differential expression of SWS1 opsin is partly due to the cis-regulatory mutation in the SWS1 gene. Specifically, the $\sim 700$ bp deletion located in the proximal promoter of $A$. baenschi might lead to the loss of putative enhancers needed to drive SWS1 expression.

Our findings indicate that the region spanning the deletion in the SWS1 promoter comprise a miRNA miR-729 and a non-coding element (CNE) that is highly conserved in nearly 250 million years of teleost evolution. Such extensive conservation points to the functional importance of both elements to SWS1 expression. Exactly how the $m i R-729$ and CNE modulate the SWS1 gene and its expression in cichlids is still unclear. miRNA's usually bind to the 3' UTR of genes and work as post-transcriptional repressors [71]. It is possible that the miR-729 prevents the misexpression of genes in the wrong cone type. For example, experimental work in mice has shown that Retinoic Acid Receptor Gamma $(R X R Y)$ suppresses S-cone opsin (homologous to SWS1 in teleosts) [72]. $R X R \gamma$ is one of the putative targets of miR-729 in cichlids. We hypothesize that in $M$. mbenji (lacking the deletion), $m i R-729$ works to keep the levels of $R X R Y$ low, so that the SWS1 cone opsin expression is not repressed. In $A$. baenschi, absence of the $m i R-729$ due to the deletion can lead to high expression of $R X R \gamma$, thereby preventing SWS1 expression. Some of the putative transcription factors that can bind to the CNE in cichlids include upstream genes well-known to be involved with photoreceptor development such as Cone Rod Homeobox (CRX) and Orthodenticle Homeobox 2 (OTX2). With A. baenschi missing these transcription factor binding sites, we postulate that its SWS1 expression will be low.

It is interesting to note that some of the predicted targets for $m i R-729$ are the same transcription factors with putative binding sites in the CNE (Table 4). All cone cells arise from the same pool of retinal progenitor cells and it has been shown that the adoption of a specific cone fate depends on the precisely timed, coordinated and often repeated expression of key transcription factors $[73,74]$. It is possible that the spatial and temporal expression of miRNA could act as a negative feedback loop and determine a certain cell-fate decision. Previous work in medaka has shown that the homologous SWS1 CNE drives the coexpression of both the miR-729 and the SWS1 opsin in the same cone cells [67]. This SWS1 cone-specific expression of $m i R-729$ in cichlids will be tested in the future with the help of reporter constructs. In order to conclusively prove that the miR-729 and CNE play a role in moderating SWS1 expression, future studies with CRISPR targets against the miR-729 and specific sites within the CNE need to be carried out.

In trying to determine whether the SWS1 promoter deletion was common, we found that only 4 of 50 species tested in Lake Malawi had the mutation. All species that had the deletion have low SWS1 levels but all species with low SWS1 expression did not have the deletion. This is not surprising because we found two other QTL that influence SWS1 opsin expression in our cross. It is possible that the differential expression of SWS1 is achieved by other mechanisms that arose in parallel in various species in Lake Malawi. Interestingly, species with the deletion were quite disparate and include two species of Aulonocara, one species of Placidochromis, and one species of Trematocranus. This suggests random sorting of the deletion into different lineages as occurs for ancestral polymorphisms. None of the species outside the lake, particularly species from Lake Victoria ( $P$. nyererei) and Tanganyika ( $N$. brichardi) and the riverine species 
(O. niloticus and $A$. burtoni) had the deletion, indicating that the deletion in the SWS1 promoter likely arose within Lake Malawi and is being shared either by hybridization or sorting through the flock as standing genetic variation [37, 75]. Future work looking at whole genomes from various species in each of these lakes will enable us to identify if other species carry the deletion.

\section{Opsin expression in cichlids is controlled by multiple, overlapping QTL}

With the exception of one cis-QTL for SWS1 on LG17, our results indicate that most opsins in cichlids are regulated by trans loci located in genomic regions distant from the opsins. Being upstream of the immediate genes regulating the trait, trans changes are often thought to be inefficient because of the widespread and often negative pleiotropic effects achieved by simultaneously affecting multiple downstream targets [76]. We speculate that in cichlids, trans mutations might actually be favored for two main reasons. First, evidence from other organisms points to the existence of Locus Control Regions (LCRs) that modulate the expression of downstream opsin tandem arrays [77]. These LCRs are usually driven by multiple trans acting factors operating as switches. By virtue of its sequestered location (LG17), SWS1 opsin need not be driven by a LCR and thus its promoter might be susceptible to accumulating cis-changes. Second, closely related species in Lake Malawi often exhibit coordinated differences in the expression of multiple opsins [78]. Such synchronized changes can be more easily achieved by a single trans change influencing multiple downstream opsins as opposed to individual cis mutations in each of the opsin promoters.

The QTL for single cone (LG14, LG20 and LG17) and double cone opsins (LG10 and LG16) are co-localized to the same chromosomal regions. Although this might be a direct outcome of the normalization procedure used in the study, previous in-situ hybridization work both in the hybrids of the MA cross as well other Malawi species have shown that in conditions of altered light spectra, both the single cone and double cone opsins are co-expressed in their respective cell types in certain parts of the retina $[63,79,80]$. Therefore, it is not unreasonable to hypothesize that at least a few loci controlling their expression might be shared as well. Co-mapping of a QTL for LWS with 2 QTLs for SWS1 and SWS2B opsins, independent of the normalization, is striking. Such genetic coupling between the single and double cone opsins hints at pleiotropic effects, although at this stage multiple linked genes cannot be ruled out. Future fine-mapping of the shared QTL interval on LG14 will help resolve this issue.
Previously, Castle-Wright estimates based on gene expression levels in the three generations of the cross $\left(\mathrm{F}_{0}\right.$, $F_{1}$ and $F_{2}$ ) suggested that $\sim 8$ loci govern differences between $S W S 1 / S W S 2 B$ opsin expression in the MA cross [63]. In the current study, we were able to detect only 3 of 8 loci for these opsins. Failure to recognize additional loci might be due to the complex architecture of the single cone opsins including multiple genes with pleiotropic and epistatic effects along with dominance. For the green-sensitive opsins in the double cones $(R H 2 A$ and $R H 2 B$ ), we were successful in identifying 3 QTL on LG10 and LG16. This was more than what had been previously predicted from the Castle-Wright estimation. The LWS opsin exhibits transgressive expression in the $\mathrm{F}_{2}$ generation of this hybrid cross, which we previously theorized was due to the presence of two loci with opposing effects in each parent [63]. Subsequent segregation of the alleles with effects in the same direction leads to $F_{2}$ offspring with high expression levels. Consistent with our hypothesis, in the present study we found 3 QTL for $L W S$ opsin with divergent effects. While the inheritance of $M$. mbenji alleles in the largest QTL on LG10 increases LWS expression, the M. mbenji alleles seem to decrease expression in the other QTL on LG14 and LG16. Failure to find the fourth QTL for $L W S$ as projected by the Castle-Wright estimator might be due to the limitations discussed above.

Together with our previously published QTL generated from the TA cross, we now have a total of 23 QTL linked to the differential expression of all six cichlid cone opsins in cichlids. Of the 23 QTL, two (RH2A and LWS on LG10) replicate between the two studies. Also, the putative QTL for $S W S 2 A$ expression on LG23 (which we discarded in this study, for reasons- see Results) matches the QTL of the same opsin from the TA cross. The common parental species between the two crosses, A. baenschi might be the source of these replicate QTL. Nevertheless, we postulate that QTL results from both the crosses can be combined for two main reasons. First, different species in Lake Malawi all diverged from a recent ancestor less than a million years old [41]. This recent evolutionary history resulted in the maintenance of a high degree of similarity between the genomes of different species. Most cichlid species have conserved genomic structure, with contiguous stretches of nearly identical sequence. Second, standing genetic variation, mostly as ancestrally-inherited polymorphisms, seems to be an important source for genetic diversity in cichlids [75]. Repeated selection of the same allele independently by different species can lead to shared genetic mechanisms. Currently, fine-mapping and identification of candidate gene (and causative mutation) in the QTLs from the TA cross is in progress in 
the lab. It will be interesting to see if the same genes can explain the variation in opsin expression in the MA cross as well.

\section{Candidate genes for differential expression of opsins}

All the trans QTL identified in this study correspond to discrete regions in the genome and contain numerous candidate genes. Although the list provided in Table 3 is drastically condensed, it is the first step towards identifying suitable candidates and also provides a point of reference for our fine-mapping efforts. There is the possibility that the differential expression of the opsins in these regions is being regulated by other factors such as microRNAs, non-coding RNAs and epigenetic elements that we have not considered here.

One of the candidate genes for $L W S$ expression is T-box Transcription Factor TBX2B, located in the QTL on LG10. In zebrafish, this gene has been implicated in promoting the production of SWS1 cone cells by repressing the rod differentiation pathway [27]. Another good candidate is NEUROD4, a cell cycle regulator gene on LG14 that promotes photoreceptor fate, while inhibiting other retinal cell fates in zebrafish [81]. Other genes connected to photoreceptor function thereby making them suitable candidates include GRK1 on LG10 (Phosphorylates the rod opsin leading to its inactivation) [82], and NR1D1 on LG20 (Affects the survival of the S-cones; mutations in this gene lead to increased sensitivity to blue light in humans) [83].

\section{Conclusions}

In this study, we have shown that both cis and trans regulatory changes are associated with the divergence in photoreceptor sensitivity in African cichlids. Additionally, we uncover a regulatory deletion in close proximity to the start site of the SWS1 opsin that likely aids its expression variation in these fishes. This is our second regulatory deletion that we have identified as possibly playing a role in cichlid opsin expression (see [84]) suggesting that indels may be an important source of variation and might contribute to cichlid explosive radiation.

Expression of SWS1 opsin in Lake Malawi cichlids is tied to their visual ecology, having been previously correlated with foraging and predatory behavior [53, 85]. Zooplanktivorous cichlids and algal browsers in Lake Malawi usually have higher SWS1 expression than species that do not rely on plankton for their nourishment. Furthermore, behavioral evidence in cichlids with SWS1 expression shows that the duration of finding prey is significantly increased under illumination conditions devoid of ultraviolet input. Finding the potential cis-regulatory deletion and other loci associated with its differential expression could thus serve as the first step towards understanding the adaptive origins of this trait.

\section{Methods \\ Genetic cross}

$\mathrm{F}_{2}$ hybrids generated from an intergeneric cross between two Lake Malawi species Aulonocara baenschi and Metriaclima mbenji (MA cross) were sampled for this study [63]. Briefly, hybrid progeny were generated from a mating between a single $A$. baenschi male and a single M. mbenji female. M. mbenji parental specimen used for the cross was third generation lab-raised progeny generated from a wild-caught stock maintained in the aquaculture facility at the University of Maryland. The $A$. baenschi parent was multiple generation progeny generated from fish obtained from the aquarium trade. A single male and ten females from the $F_{1}$ generation were randomly chosen and then mated to generate $350 \mathrm{~F}_{2}$ progeny $\left(\sim 40 \mathrm{~F}_{2}\right.$ families). All individuals used in this study were sexually mature ( $>6$ months of age) and raised under laboratory conditions. Sampling was done randomly with respect to sex. Following euthanasia with a lethal dose of buffered MS-222, retinas were dissected and stored in RNAlater at -20C.

\section{Phenotyping opsin expression}

Opsin expression in the $\mathrm{F}_{2}$ hybrids was quantified as described previously [63]. RNA was extracted from dissected retinas and transcribed into CDNA with Invitrogen Superscript III (Invitrogen, NY, USA) following manufacturer instructions. Relative opsin expression from each cDNA sample was measured by real-time, quantitative PCR (RT-PCR) using opsin specific Taqman primers and probes on a Roche Lightcycler 480 according to previously published protocols [47, 86, 87] ( $R H 2 A \alpha$ and $R H 2 A \beta$ genes that are more than $99 \%$ identical were combined and noted as $R H 2 A$ ). Gene expression was normalized as a percent of total opsin expression within single cones and double cones respectively. Therefore, the fraction, $f$, of $S W S 1$ expression in the single cones is calculated as:

$$
\mathrm{f}_{S W S 1, \mathrm{SC}}=\mathrm{T}_{S W S 1} /\left(\mathrm{T}_{S W S 1}+\mathrm{T}_{S W S 2 B}+\mathrm{T}_{S W S 2 A}\right)
$$

where $\mathrm{T}$ is the transcript level for each of the single cone opsin genes. Similarly, the fraction of LWS opsin expression in double cones is:

$$
\mathrm{f}_{L W S, \mathrm{DC}}=\mathrm{T}_{L W S} /\left(\mathrm{T}_{R H 2 A}+\mathrm{T}_{R H 2 B}+\mathrm{T}_{L W S}\right)
$$

\section{RAD-Seq libraries}

To identify SNPs for QTL mapping, restriction-site associated DNA sequencing (RAD-Seq) was performed on $\mathrm{F}_{2}$ progeny. Reduced representation libraries were constructed by Floragenex Inc. (Portland, OR, USA) according to the protocol of Baird et al. [88]. High-molecular weight genomic DNA was extracted from fin clips of 
$157 \mathrm{~F}_{2}$ hybrids and the $2 \mathrm{~F}_{0}$ parents using DNeasy blood and tissue kits (Qiagen Inc. CA, USA) and quantified on a BioTek FLX800 fluorometer (BioTek, VT, USA) with Quant-iT dsDNA High-sensitivity kit (Invitrogen, NY, USA). $20 \mathrm{ng} / \mathrm{ul}$ DNA from each sample was digested with HF-Sbfi restriction enzyme, ligated to 95 unique barcode linkers and combined into two libraries (the second library had $66 \mathrm{~F}_{2}$ and the two $\mathrm{F}_{0}$ individuals). Each library was then randomly sheared to an average size of $500 \mathrm{bp}$ and size selected between 300 and $500 \mathrm{bp}$. Following ligation with Illumina adapters and PCR enrichment, both libraries were gel purified and quantified, before being sequenced single-end on an Illumina Hi-Seq 2000 at the University of Oregon Genomics Core Facility.

Raw sequence reads were filtered for quality using the FASTX toolkit. In silico analysis for identification of SNPs and determination of genotypes was performed with the built-in wrapper program denovo_map.pl in Stacks (version 1.35) as described by Catchen et al. [89]. Briefly, demultiplexed reads of each individual were assembled into unique loci and heterozygous alleles were identified (allowing for a maximum of 1 mismatch). Then, orthologous stacks from the $F_{0}$ parents were matched into a common catalog of loci and differentially fixed SNPs were identified between them. Lastly, the $F_{2}$ stacks were compared to the parental stack catalog to infer genotypes at each locus. Conservative criteria were imposed for inferring genotypes from stack loci. Default parameters were modified to require the minimum number of reads to call homozygous genotype in the $F_{2}$ to be 10 (min_hom_seqs), the minor allele frequency below which a stack is deemed a homozygote to be $1 / 15$ or 0.066 (min_het_seqs); the minimum frequency of minor allele to be called a heterozygote to be $2 / 15$ or 0.133 (max_het_seqs). Genotypes with minor allele depth between $0.066-0.133$ were considered to not have adequate support and were deemed 'unknown'. The RAD loci were filtered further to exclude markers that were not differentially fixed between the $F_{0}$ parents. Finally, the consensus sequence of each RAD marker was aligned to the M_zebra_UMD1_genome assembly using BLAST [90]. Only markers with unique hits in the $M$. zebra genome were included for consequent analyses.

\section{Linkage map construction}

Genetic linkage mapping was performed in R/qtl ( $R$ Foundation for Statistical Computing) following the guidelines described in Broman and Sen [91]. Markers were excluded from subsequent analyses if they were not genotyped in at least $90 \%$ of the individuals or failed to satisfy Hardy-Weinberg expectations (after correcting for multiple comparisons). All the RAD-Seq markers were grouped into linkage groups by requiring a maximum recombination frequency of 0.35 and a minimum $\log _{10}$ odds ratio (LOD) threshold of 10. Within each linkage group, markers were then ordered by repeatedly rippling the order of a 7-marker sliding window, ultimately choosing the order with the fewest obligate crossovers. Large-scale changes in marker order were made manually and markers that did not fall into linkage groups were dropped from the analysis. A LOD score for each genotype was calculated in order to identify statistically unlikely events, such as closely placed double crossovers. After excluding such incorrect genotypes, final intermarker distances were estimated using the Kosambi map function [92]. Lastly, linkage groups (LGs) in the constructed map were aligned to the anchored O. niloticus (O_niloticus_UMD1) genome assembly to assign to the correct cichlid LG nomenclature [93].

\section{QTL analyses}

Quantitative trait loci (QTL) regulating opsin expression were detected following methods in O'Quin, et al. [62]. QTL analyses were performed in R/qtl using the fully automated model selection algorithm Stepwise that searches for multiple interacting QTL for each opsin [91]. With the stepwise analysis, LOD of association between opsin expression and the genotypes at every marker were computed using forward selection to a model with 10 QTL, followed by backward elimination to the null model of no QTL. Missing genotype data at the putative QTL were taken into account by using Haley Knot regression. The function fitgtl was used to calculate the LOD score and percentage of variance in opsin expression explained by each QTL. Significance thresholds for the QTL were determined from the 95th percentile of the genome-wide LOD scores calculated from 1000 permutations. Markers with the highest LOD score were used to test the effect of each QTL. Interval estimates of the location of the QTL were obtained from 95\% Bayes credible intervals (BI) extended to the nearest genotyped markers. For each QTL, candidate genes were estimated by matching the markers flanking the Bayes intervals (BI) to their corresponding regions in the $O$. niloticus (O_niloticus_UMD1) genome [93].

\section{SWS1 coding sequence analysis}

SWS1 coding sequences from M. mbenji, A. baenschi and $M$. zebra were obtained from Genbank (Accession numbers: M. mbenji: HM049223.1, A. baenschi: GQ422525.1, M. zebra: NM_001310074.1). Following translation using the Expasy Translate tool, amino acid sequences from all three species were aligned along with bovine rhodopsin (UniProtKB P02699) using ClustalW (version 2) to identify amino acid substitutions that might be functionally relevant [94]. 
Attention was paid to sites where the substitution altered amino acid polarity, specifically in the retinal binding pocket of the opsin protein, similar to previous methods [69].

\section{SWS1 promoter sequencing}

The promoter of the SWS1 gene was Sanger sequenced with overlapping primer sets in both the $F_{0}$ of the cross as well as in a panel of 17 species from Lake Malawi with previously characterized opsin expression profiles. Retinas of the 17-species panel were collected during a field expedition to southern Lake Malawi in 2008 [78]. The primers were designed based on M. zebra genome (Primer sequences provided in Additional file 3: Table S1). DNA from each sample was PCR amplified, size selected by gel-electrophoresis, and sequenced using Applied Biosystems Big Dye Terminators on a ABI 3730xl. For each species, sequences from all the primers were assembled and edited manually in Geneious (version 11.0.5) before making comparisons [95]. The SWS1 promoter sequence alignment file for the two parental species of the cross and the 17-species panel has been submitted to Genbank (Accession numbers: MH122659, MH122660 and MH215441 to MH215457). Since sequencing showed that the deletion was identical across species, 29 additional Lake Malawi species (with known opsin expression) were genotyped for the presence or absence of the deletion by fragment size on an agarose gel [84]. Further, whole genomes from 14 species native to the Malawi catchment area, were obtained from the Wellcome Sanger Institute [65] (12 of the Wellcome genome species are also included in the 29 species Malawi panel, and 2 species- Aulonocara stuartgranti and Astatotilapia calliptera are not). Sequence data from each species was aligned to the M. zebra reference assembly before looking for differences in the SWS1 promoter. Finally, previously published genomes of cichlids archetypal of Lake Tanganyika (Neolamprologous brichardi), Lake Victoria (Pundamilia nyererei) and two ancestral riverine species (Astatotilapia burtoni and Oreochromis niloticus) were also examined to look for the deletion in the SWS1 promoter [42]. All sampled species along with their single cone opsin expression profiles are provided in Additional file 5.

\section{Phylogenetic footprinting assay}

DNA spanning from the last exon of TNPO3 (the gene upstream of SWS1) to the first few exons of the SWS1 gene were obtained from the M. zebra genome assembly (LG17: 12796067-12,802,659). Orthologous DNA regions spanning the same interval were obtained from the genome assemblies of four other teleost fish- Tilapia (Oreochromis niloticus, Tilapia_Broad assembly (LG17: 11751000-11,756,599), medaka (Oryzias latipes, NIG/UT MEDAKA1/oryLat2 assembly, scaffold1021: 45422-53,836), stickleback (Gasterosteus aculeatus, Broad/gasAcul assembly, chrUN: 2226859522,274,962) and zebrafish (Danio rerio, GRCz10/danRer10 assembly, chr4: 13578911-13,588,818). Comparative sequence analysis was implemented in mVista (accessible at http://www-gsd.lbl.gov/vista/) to perform pairwise alignments of orthologous DNA sequences from multiple species [96]. Sequences were aligned with the global alignment tool MLAGAN and regions were deemed as conserved elements if they showed $50 \%$ identity within a $50 \mathrm{bp}$ window for all species except zebrafish [97]. Since the divergence time between M. zebra and zebrafish is nearly twice as much (230 million years) as that of its next distant relative- stickleback (117 million years), the conservation parameters in zebrafish were relaxed and set to $25 \%$ identity within the same $50 \mathrm{bp}$ interval ( $M$. zebra- medaka: 98 million years, and M. zebraTilapia: 25 million years) [98].

Transcription factor binding site (TFBS) analysis on the CNE in the promoter of the SWS1 opsin was performed using the JASPAR CORE Vertebrata 2016 database [99]. Settings were adjusted to shortlist putative transcription factor binding profiles with a relative score of $\geq 0.80$. The identity of the microRNA (miRNA) was established using mirBase [100]. Potential target genes for the miRNA were predicted using the computational algorithm miRanda according to the methods described in Enright et al. [101]. 3' UTR FASTA sequences for all cichlid genes were obtained from the Tilapia Broad assembly.

\section{Additional files}

Additional file 1: RAD-Sequencing processing statistics for $F_{0}$ and $F_{2}$ in the cross. (XLSX $56 \mathrm{~kb}$ )

Additional file 2: FASTA sequences of the 1217 RAD markers for the construction of linkage map and QTL analysis. Their positions in the cichlid genome are also provided. (XLSX $125 \mathrm{~kb}$ )

Additional file 3: Figures for the linkage map used for QTL analysis and effect plots for single and double cone opsin QTL are provided. Also sequencing primers used for the promoter of SWS1 opsin are also provided. (PDF $284 \mathrm{~kb}$ )

Additional file 4: Linkage map along with phenotype and genotype data used for the QTL analysis. Relative opsin expression (in percentage) of all $F_{2}$ individuals used for the QTL study is provided. Opsin expressed is measured relative to total opsin expression within single cones and double cones respectively. The genotypes of all individuals at every marker are also denoted. Linkage positions of all markers used in the study are also provided. (XLSX $752 \mathrm{~kb}$ )

Additional file 5: All species tested for the presence or absence of deletion in the promoter of the SWS1 opsin. This list includes the parental species of the cross, sampled species from Lake Malawi, and species from the Wellcome and Broad genomes. Opsin expression expressed as percentage of total opsin expression in single cones is provided for each species. (XLSX $49 \mathrm{~kb}$ )

Additional file 6: Alignment of the zebrafish dre-mi-729 with teleost sequences in the region upstream of SWS1 opsin. Highly conserved nucleotide positions are indicated in green. The predicted cichlid miRNA is highly conserved with zebrafish miRNA dre-mi-729. (PDF $262 \mathrm{~kb}$ ) 


\section{Abbreviations}

BI: Bayes interval; CNE: Conserved non-coding element; LCR: Locus control region; LG: Linkage group; LOD: Logarithm of odds; MA cross: Metriaclima mbenji and Aulonocara baenschi cross; miRNA: microRNA;

MSP: Microspectrophotometry; PVE: Percent variance in expression; QTL: Quantitative trait locus; RAD-Seq: Restriction-site associated DNA sequencing; SNP: Single-nucleotide polymorphism; TA cross: Tramitochromis intermedius and Aulonocara baenschi cross; TFBS: Transcription factor binding site; UTR: Untranslated region

\section{Acknowledgements}

We thank Kelly O'Quin for assistance with map construction and QTL analysis. We thank Marissa Schoepp for helping with the retina dissections. We thank Daniel Escobar-Camacho for helping with figures, Miranda Yourick and Benjamin Sandkam for valuable suggestions on the manuscript.

\section{Funding}

This work was supported by the National Eye Institute at the National Institutes of Health (NIH R01EY024639 to K.L.C).

\section{Availability of data and materials}

All data generated or analyzed during this study are included in this published article [and its additional information files]. All sequence data has been submitted to Genbank (Accession numbers provided in the Methods). Any other information can be obtained from the corresponding author on reasonable request.

\section{Authors' contributions}

SPN generated the hybrid cross, performed the QTL experiment and analyses, and drafted the manuscript. MAC performed the bioinformatic analysis pertaining to the study and reviewed the manuscript. KLC conceptualized the study and critically reviewed the article. All authors read and approved the final manuscript.

\section{Ethics approval}

All animal procedures were approved by the University of Maryland College Park IACUC protocol R-09-73 and R12-90.

\section{Consent for publication}

Not applicable

\section{Competing interests}

The authors declare that they have no competing interests.

\section{Publisher's Note}

Springer Nature remains neutral with regard to jurisdictional claims in published maps and institutional affiliations.

Received: 7 March 2018 Accepted: 28 November 2018 Published online: 18 December 2018

\section{References}

1. Gompel N, Prud'homme B, Wittkopp PJ, Kassner VA, Carroll SB. Chance caught on the wing: cis-regulatory evolution and the origin of pigment patterns in Drosophila. Nature. 2005:433(7025):481-7.

2. Jeong S, Rebeiz M, Andolfatto P, Werner T, True J, Carroll SB. The evolution of gene regulation underlies a morphological difference between two Drosophila sister species. Cell. 2008;132(5):783-93.

3. Gruber JD, Vogel K, Kalay G, Wittkopp PJ. Contrasting properties of genespecific regulatory, coding, and copy number mutations in Saccharomyces cerevisiae: frequency, effects, and dominance. PLoS Genet. 2012;8(2): e1002497.

4. Tirosh I, Reikhav S, Levy AA, Barkai N. A yeast hybrid provides insight into the evolution of gene expression regulation. Science. 2009;324(5927):659-62.

5. Yvert G, Brem RB, Whittle J, Akey JM, Foss E, Smith EN, Mackelprang R, Kruglyak L. Trans-acting regulatory variation in Saccharomyces cerevisiae and the role of transcription factors. Nat Genet. 2003;35(1):57-64.

6. Wray GA. The evolutionary significance of cis-regulatory mutations. Nat Rev Genet. 2007:8(3):206-16.

7. Hoekstra HE, Coyne JA. The locus of evolution: evo devo and the genetics of adaptation. Evolution. 2007;61(5):995-1016.
8. Stern DL, Orgogozo V. The loci of evolution: how predictable is genetic evolution? Evolution. 2008;62(9):2155-77.

9. Carroll SB. Evo-devo and an expanding evolutionary synthesis: a genetic theory of morphological evolution. Cell. 2008;134(1):25-36.

10. Wittkopp PJ, Haerum BK, Clark AG. Evolutionary changes in cis and trans gene regulation. Nature. 2004;430(6995):85-8.

11. Wang D, Sung HM, Wang TY, Huang C, Yang P, Chang T, Wang YC, Tseng $\mathrm{DL}, \mathrm{Wu} J \mathrm{JP}$, Lee $\mathrm{TC}$, et al. Expression evolution in yeast genes of single-input modules is mainly due to changes in trans-acting factors. Genome Res. 2007:17(8):1161-9.

12. Wittkopp PJ, Haerum BK, Clark AG. Regulatory changes underlying expression differences within and between Drosophila species. Nat Genet. 2008;40(3):346-50.

13. Domyan ET, Kronenberg Z, Infante CR, Vickrey Al, Stringham SA, Bruders R, Guernsey MW, Park S, Payne J, Beckstead RB, et al. Molecular shifts in limb identity underlie development of feathered feet in two domestic avian species. Elife. 2016;5:e12115.

14. Shapiro MD, Bell MA, Kingsley DM. Parallel genetic origins of pelvic reduction in vertebrates. Proc Natl Acad Sci U S A. 2006;103(37):13753-8.

15. McGaugh SE, Gross JB, Aken B, Blin M, Borowsky R, Chalopin D, Hinaux H, Jeffery WR, Keene A, Ma L, et al. The cavefish genome reveals candidate genes for eye loss. Nat Commun. 2014;5:5307.

16. Linnen $C R$, Kingsley EP, Jensen JD, Hoekstra HE. On the origin and spread of an adaptive allele in deer mice. Science. 2009;325(5944):1095-8.

17. Bhattacharyya MK, Smith AM, Ellis TN, Hedley C, Martin C. The wrinkled-seed character of pea described by Mendel is caused by a transposon-like insertion in a gene encoding starch-branching enzyme. Cell. 1990;60:115-22.

18. Yokoyama S. Molecular evolution of vertebrate visual pigments. Prog Retin Eye Res. 2000;19(4):385-419.

19. Davies WI, Collin SP, Hunt DM. Molecular ecology and adaptation of visual photopigments in craniates. Mol Ecol. 2012;21(13):3121-58.

20. Hunt DM, Bowmaker JK, Cowing JA, Carvalho LDS, Parry JWL, Wilkie SE, Davies WL. Spectral tuning of vertebrate visual pigments. Perception. 2006;35:167.

21. Hunt DM, Carvalho LS, Cowing JA, Davies WL. Evolution and spectral tuning of visual pigments in birds and mammals. Philos Trans R Soc Lond Ser B Biol Sci. 2009;364(1531):2941-55.

22. Adler R, Raymond PA. Have we achieved a unified model of photoreceptor cell fate specification in vertebrates? Brain Res. 2008;1192:134-50.

23. Swaroop A, Kim D, Forrest D. Transcriptional regulation of photoreceptor development and homeostasis in the mammalian retina. Nat Rev Neurosci. 2010;11(8):563-76.

24. Carroll J, Rossi EA, Porter J, Neitz J, Roorda A, Williams DR, Neitz M. Deletion of the X-linked opsin gene array locus control region (LCR) results in disruption of the cone mosaic. Vis Res. 2010;50(19):1989-99.

25. Ng L, Hurley JB, Dierks B, Srinivas M, Salto C, Vennstrom B, Reh TA, Forrest D. A thyroid hormone receptor that is required for the development of green cone photoreceptors. Nat Genet. 2001;27(1):94-8.

26. Chinen A, Hamaoka T, Yamada Y, Kawamura S. Gene duplication and spectral diversification of cone visual pigments of zebrafish. Genetics. 2003; 163(2):663-75.

27. Alvarez-Delfin K, Morris AC, Snelson CD, Gamse JT, Gupta T, Marlow FL, Mullins MC, Burgess HA, Granato M, Fadool JM. Tbx2b is required for ultraviolet photoreceptor cell specification during zebrafish retinal development. Proc Natl Acad Sci U S A. 2009;106(6):2023-8.

28. Raine JC, Hawryshyn CW. Changes in thyroid hormone reception precede SWS1 opsin downregulation in trout retina. J Exp Biol. 2009;212(17):2781-8.

29. Kocher T. Adaptive evolution and explosive speciation: the cichlid fish model. Nat Rev Genet. 2004;5(4):288-98.

30. Seehausen $\mathrm{O}$. African cichlid fish: a model system in adaptive radiation research. Proc R Soc B Biol Sci. 2006;273(1597):1987-98.

31. Salzburger $\mathbf{W}$. The interaction of sexually and naturally selected traits in the adaptive radiations of cichlid fishes. Mol Ecol. 2009;18(2):169-85.

32. Fryer G, lles TD. The Cichlid Fishes of the Great Lakes of Africa. Their Biology and Evolution. Oliver and Boyd, Edinburgh. 1972. p. 641.

33. Kornfield I, Smith P. African cichlid fishes: Model systems for evolutionary biology. Annu Rev Ecol Syst. 2000;31:163.

34. Danley P, Kocher T. Speciation in rapidly diverging systems: lessons from Lake Malawi. Mol Ecol. 2001;10(5):1075-86.

35. Turner $G$, Seehausen $O$, Knight M, Allender $C$, Robinson R. How many species of cichlid fishes are there in African lakes? Mol Ecol. 2001;10(3):793-806. 
36. Albertson R, Markert J, Danley P, Kocher T. Phylogeny of a rapidly evolving clade: the cichlid fishes of Lake Malawi, East Africa. Proc Natl Acad Sci U S A 1999;96(9):5107-10.

37. Joyce DA, Lunt DH, Genner MJ, Turner GF, Bills R, Seehausen O. Repeated colonization and hybridization in Lake Malawi cichlids. Curr Biol. 2011;21(3): R108-9.

38. Albertson R, Streelman J, Kocher T. Genetic basis of adaptive shape differences in the cichlid head. J Hered. 2003:94(4):291-301.

39. Carleton K, Hofmann C, Klisz C, Patel Z, Chircus L, Simenauer L, Soodoo N, Albertson R, Ser J. Genetic basis of differential opsin gene expression in cichlid fishes. J Evol Biol. 2010;23(4):840-53.

40. Parnell NF, Streelman JT. Genetic interactions controlling sex and color establish the potential for sexual conflict in Lake Malawi cichlid fishes. Heredity (Edinb). 2013;110(3):239-46.

41. Genner M, Seehausen O, Lunt D, Joyce D, Shaw P, Carvalho G, Turner G. Age of cichlids: new dates for ancient lake fish radiations. Mol Biol Evol. 2007;24(5):1269-82.

42. Brawand D, Wagner C, Li Y, Malinsky M, Keller I, Fan S, Simakov O, Ng A, Lim $Z$, Bezault $E$, et al. The genomic substrate for adaptive radiation in African cichlid fish. Nature. 2014;513(7518):375.

43. Albertson R, Streelman J, Kocher T. Directional selection has shaped the oral jaws of Lake Malawi cichlid fishes. Proc Natl Acad Sci U S A. 2003; 100(9):5252-7.

44. Roberts RB, Ser JR, Kocher TD. Sexual conflict resolved by invasion of a novel sex determiner in Lake Malawi cichlid fishes. Science. 2009:326(5955):998-1001.

45. O'Quin C, Drilea A, Roberts R, Kocher T. A small number of genes underlie male pigmentation traits in Lake Malawi cichlid fishes. J Exp Zool B Mol Dev Evol. 2012;318B(3):199-208.

46. York RA, Patil C, Hulsey CD, Anoruo O, Streelman JT. Fernald RD: Evolution of bower building in Lake Malawi cichlid fish: phylogeny, morphology, and behavior. Front Ecol Evol. 2015;3:18.

47. Spady T, Parry J, Robinson P, Hunt D, Bowmaker J, Carleton K. Evolution of the cichlid visual palette through ontogenetic subfunctionalization of the opsin gene arrays. Mol Biol Evol. 2006;23(8):1538-47.

48. Lee B, Lee W, Streelman J, Carleton K, Howe A, Hulata G, Slettan A, Stern J, Terai $Y$, Kocher T. A second-generation genetic linkage map of tilapia (Oreochromis spp.). Genetics. 2005;170(1):237-44.

49. Carleton $\mathrm{K}$, Kocher T. Cone opsin genes of African cichlid fishes: tuning spectral sensitivity by differential gene expression. Mol Biol Evol. 2001;18(8): 1540-50.

50. Parry J, Carleton K, Spady T, Carboo A, Hunt D, Bowmaker J. Mix and match color vision: tuning spectral sensitivity by differential opsin gene expression in Lake Malawi cichlids. Curr Biol. 2005;15(19):1734-9.

51. Spady T, Seehausen O, Loew E, Jordan R, Kocher T, Carleton K. Adaptive molecular evolution in the opsin genes of rapidly speciating cichlid species. Mol Biol Evol. 2005;22(6):1412-22.

52. Carleton K. Cichlid fish visual systems: mechanisms of spectral tuning. Integr Zool. 2009;4(1):75-86.

53. Jordan R, Howe D, Juanes F, Stauffer J, Loew E. Ultraviolet radiation enhances zooplanktivory rate in ultraviolet sensitive cichlids. Afr J Ecol. 2004; 42(3):228-31.

54. Dijkstra P, Seehausen O, Groothuis T. Direct male-male competition can facilitate invasion of new colour types in Lake Victoria cichlids. Behav Ecol Sociobiol. 2005;58(2):136-43.

55. Dobberfuhl A, Ullmann J, Shumway C. Visual acuity, environmental complexity, and social organization in African cichlid fishes. Behav Neurosci. 2005;119(6):1648-55.

56. Kidd M, Danley P, Kocher T. A direct assay of female choice in cichlids: all the eggs in one basket. J Fish Biol. 2006;68(2):373-84

57. Dijkstra P, Seehausen O, Pierotti M, Groothuis T. Male-male competition and speciation: aggression bias towards differently coloured rivals varies between stages of speciation in a Lake Victoria cichlid species complex. J Evol Biol. 2007;20(2):496-502

58. Seehausen $\mathrm{O}$, van Alphen J. The effect of male coloration on female mate choice in closely related Lake Victoria cichlids (Haplochromis nyererei complex). Behav Ecol Sociobiol. 1998;42(1):1-8.

59. Selz O, Pierotti M, Maan M, Schmid C, Seehausen O. Female preference for male color is necessary and sufficient for assortative mating in 2 cichlid sister species. Behav Ecol. 2014;25(3):612-626.

60. Maan M, Hofker K, van Alphen J, Seehausen O. Sensory drive in cichlid speciation. Am Nat. 2006;167(6):947-54.
61. Seehausen O, Terai Y, Magalhaes I, Carleton K, Mrosso H, Miyagi R, van der Sluijs I, Schneider M, Maan M, Tachida H, et al. Speciation through sensory drive in cichlid fish. Nature. 2008;455(7213):620-U623.

62. O'Quin K, Schulte J, Patel Z, Kahn N, Naseer Z, Wang H, Conte M, Carleton K. Evolution of cichlid vision via trans-regulatory divergence. BMC Evol Biol. 2012;12:251.

63. Nandamuri S, Dalton B, Carleton K. Determination of the genetic architecture underlying shortwavelength sensitivity in Lake Malawi cichlids. Hered. 2017:379-390.

64. Albertson RC, Powder KE, Hu Y, Coyle KP, Roberts RB, Parsons KJ. Genetic basis of continuous variation in the levels and modular inheritance of pigmentation in cichlid fishes. Mol Ecol. 2014;23(21):5135-50.

65. Malinsky M, Svardal H, Tyers AM, Miska EA, Genner MJ, Turner GF, Durbin R. Whole genome sequences of Malawi cichlids reveal multiple radiations interconnected by gene flow. Nat Ecol Evol. 2018;2(12):1940-1955.

66. Kloosterman WP, Steiner FA, Berezikov E, de Bruijn E, van de Belt J, Verheul $\mathrm{M}$, Cuppen E, Plasterk RH. Cloning and expression of new microRNAs from zebrafish. Nucleic Acids Res. 2006;34(9):2558-69.

67. Daido $Y$, Hamanishi S, Kusakabe TG. Transcriptional co-regulation of evolutionarily conserved microRNA/cone opsin gene pairs: implications for photoreceptor subtype specification. Dev Biol. 2014:392(1):117-29.

68. Beavis WD. QTL analyses:power, precision, and accuracy. In: Molecular dissection of complex traits. Boca Raton: CRC Press; 1998. p. 145-62.

69. Smith A, Carleton K. Allelic variation in Malawi cichlid opsins: a tale of two genera. J Mol Evol. 2010;70(6):593-604.

70. Hauser FE, van Hazel I, Chang BS. Spectral tuning in vertebrate short wavelength-sensitive 1 (SWS1) visual pigments: can wavelength sensitivity be inferred from sequence data? J Exp Zool B Mol Dev Evol. 2014;322(7):529-39.

71. Chen K, Rajewsky N. The evolution of gene regulation by transcription factors and microRNAs. Nat Rev Genet. 2007:8(2):93-103.

72. Roberts M, Hendrickson A, McGuire C, Reh T. Retinoid X receptor gamma is necessary to establish the S-opsin gradient in cone photoreceptors of the developing mouse retina. Invest Ophthalmol Vis Sci. 2005;46(8):2897-904.

73. Turner DL, Snyder EY, Cepko CL. Lineage-independent determination of cell type in the embryonic mouse retina. Neuron. 1990;4(6):833-45.

74. Cayouette M, Poggi L, Harris WA. Lineage in the vertebrate retina. Trends Neurosci. 2006;29(10):563-70

75. Loh Y, Bezault E, Muenzel F, Roberts R, Swofford R, Barluenga M, Kidd C, Howe A, Di Palma F, Lindblad-Toh K, et al. Origins of shared genetic variation in African cichlids. Mol Biol Evol. 2013:30(4):906-17.

76. Wittkopp PJ. Genomic sources of regulatory variation in cis and in trans. Cell Mol Life Sci. 2005;62(16):1779-83.

77. Tsujimura T, Chinen A, Kawamura S. Identification of a locus control region for quadruplicated green-sensitive opsin genes in zebrafish. Proc Natl Acad Sci U S A. 2007:104(31):12813-8.

78. Hofmann C, O'Quin K, Marshall N, Cronin T, Seehausen O, Carleton K. The eyes have it: regulatory and structural changes both underlie cichlid visual pigment diversity. PLoS Biol. 2009;7(12):31000266.

79. Dalton B, Loew E, Cronin T, Carleton K. Spectral tuning by opsin coexpression in retinal regions that view different parts of the visual field. Proc R Soc B Biol Sci. 2014:281(1797):20141980.

80. Dalton B, Lu J, Leips J, Cronin T, Carleton K. Variable light environments induce plastic spectral tuning by regional opsin coexpression in the African cichlid fish. Mol Ecol. 2015;24(16):4193-204.

81. Ochocinska MJ, Hitchcock PF. NeuroD regulates proliferation of photoreceptor progenitors in the retina of the zebrafish. Mech Dev. 2009; 126(3-4):128-41.

82. Wada Y, Sugiyama J, Okano T, Fukada Y. GRK1 and GRK7: unique cellular distribution and widely different activities of opsin phosphorylation in the zebrafish rods and cones. J Neurochem. 2006;98(3):824-37.

83. Mollema NJ, Yuan Y, Jelcick AS, Sachs AJ, von Alpen D, Schorderet D, Escher P. Haider NB. Nuclear receptor rev-erb alpha (Nr1d1) functions in concert with $\mathrm{Nr2e} 3$ to regulate transcriptional networks in the retina. PLoS One. 2011;6(3):e17494

84. Schulte J, O'Brien C, Conte M, O'Quin K, Carleton K. Interspecific variation in Rx1 expression controls opsin expression and causes visual system diversity in African cichlid fishes. Mol Biol Evol. 2014;31(9):2297-308.

85. O'Quin K, Hofmann C, Hofmann H, Carleton K. Parallel evolution of opsin gene expression in African cichlid fishes. Mol Biol Evol. 2010;27(12):2839-54.

86. Hofmann C, O'Quin K, Smith A, Carleton K. Plasticity of opsin gene expression in cichlids from Lake Malawi. Mol Ecol. 2010;19(10):2064-74. 
87. Carleton KL. Quantification of transcript levels with quantitative RT-PCR. Methods Mol Biol. 2011;772:279-95.

88. Baird NA, Etter PD, Atwood TS, Currey MC, Shiver AL, Lewis ZA, Selker EU, Cresko WA, Johnson EA. Rapid SNP discovery and genetic mapping using sequenced RAD markers. PLoS One. 2008;3(10):e3376.

89. Catchen J, Amores A, Hohenlohe P, Cresko W, Postlethwait J. Stacks: building and genotyping loci de novo from short-read sequences. G3Genes Genomes Genet. 2011;1(3):171-82.

90. Conte MA, Kocher TD. An improved genome reference for the African cichlid. BMC Genomics. 2015;16:724.

91. Broman KW, Sen S. Guide to QTL mapping with R/qtl. NewYork: SpringerVerlag. Stat Biol Health. 2009:1-396.

92. Kosambi DD. The Estimation of Map Distances from Recombination Values In: Ramaswamy R, editor. DD Kosambi: Selected Works in Mathematics and Statistics. New Delhi: Springer India; 2016. p. 125-30.

93. Conte MA, Gammerdinger WJ, Bartie KL, Penman DJ, Kocher TD. A high quality assembly of the Nile Tilapia (Oreochromis niloticus) genome reveals the structure of two sex determination regions. BMC Genomics. 2017;18(1):341.

94. Gasteiger E, Gattiker A, Hoogland C, Ivanyi I, Appel RD, Bairoch A. ExPASy: the proteomics server for in-depth protein knowledge and analysis. Nucleic Acids Res. 2003:31(13):3784-8.

95. Kearse M, Moir R, Wilson A, Stones-Havas S, Cheung M, Sturrock S, Buxton S, Cooper A, Markowitz S, Duran C, et al. Geneious basic: an integrated and extendable desktop software platform for the organization and analysis of sequence data. Bioinformatics. 2012;28(12):1647-9.

96. Frazer KA, Pachter L, Poliakov A, Rubin EM, Dubchak I. VISTA: computational tools for comparative genomics. Nucleic Acids Res. 2004;32(Web Server issue):W273-9.

97. Brudno M, Do CB, Cooper GM, Kim MF, Davydov E, Program NCS, Green ED, Sidow A, Batzoglou S. LAGAN and Multi-LAGAN: efficient tools for largescale multiple alignment of genomic DNA. Genome Res. 2003;13(4):721-31.

98. Kumar S, Stecher G, Suleski M, Hedges SB. TimeTree: a resource for timelines, timetrees, and divergence times. Mol Biol Evol. 2017;34(7):1812-9.

99. Mathelier A, Zhao X, Zhang AW, Parcy F, Worsley-Hunt R, Arenillas DJ, Buchman S, Chen CY, Chou A, lenasescu H, et al. JASPAR 2014: an extensively expanded and updated open-access database of transcription factor binding profiles. Nucleic Acids Res. 2014;42(Database issue):D142-7.

100. Kozomara A, Griffiths-Jones S. miRBase: annotating high confidence microRNAs using deep sequencing data. Nucleic Acids Res. 2014; 42(Database issue):D68-73.

101. Enright AJ, John B, Gaul U, Tuschl T, Sander C, Marks DS. MicroRNA targets in Drosophila. Genome Biol. 2003;5(1):R1.

102. Blake JA, Ziman MR. Pax genes: regulators of lineage specification and progenitor cell maintenance. Development. 2014;141(4):737-51.

103. Ratscho N, Scholten A, Koch KW. Expression profiles of three novel sensory guanylate cyclases and guanylate cyclase-activating proteins in the zebrafish retina. Biochim Biophys Acta. 2009;1793(6):1110-4.

Ready to submit your research? Choose BMC and benefit from:

- fast, convenient online submission

- thorough peer review by experienced researchers in your field

- rapid publication on acceptance

- support for research data, including large and complex data types

- gold Open Access which fosters wider collaboration and increased citations

- maximum visibility for your research: over $100 \mathrm{M}$ website views per year

At $\mathrm{BMC}$, research is always in progress.

Learn more biomedcentral.com/submissions 